\title{
Optical Properties and Characterization of Prepared Sn-Doped PbSe Thin Film
}

\author{
M. R. Khanlary ${ }^{1,2}$ and E. Salavati2 \\ ${ }^{1}$ Physics Department, Imam Khomeini International University, Qazvin, Iran \\ ${ }^{2}$ Physics Department, Islamic Azad University, Qom Branch, Qom, Iran
}

Correspondence should be addressed to M. R. Khanlary, khanlary@yahoo.com

Received 10 January 2012; Revised 2 March 2012; Accepted 23 March 2012

Academic Editor: Mohindar S. Seehra

Copyright ( $) 2012$ M. R. Khanlary and E. Salavati. This is an open access article distributed under the Creative Commons Attribution License, which permits unrestricted use, distribution, and reproduction in any medium, provided the original work is properly cited.

\begin{abstract}
Physical vapor deposition of tin-doped lead selenide $(\mathrm{Sn} / \mathrm{PbSe})$ thin films on $\mathrm{SiO}_{2}$ glass is described. Interaction of high-energy $\mathrm{Ar}^{+}$ions bombardment on the doped $\mathrm{PbSe}$ films is discussed by XRD analysis. The improvement of optical band gap of $\mathrm{Sn} / \mathrm{PbSe}$ films irradiated by different doses of irradiation was studied using transmission spectroscopy.
\end{abstract}

\section{Introduction}

Lead chalcogenides $(\mathrm{PbE}, \mathrm{E}=\mathrm{S}, \mathrm{Se}, \mathrm{Te})$ are a special class of IV-VI narrow-band-gap (0.2-0.4 ev) semiconductors [1]. These materials were studied for many decades, but they still reveal some interesting properties, for example, high temperature of continuous-wave photoluminescence, which are able to improve mid infrared lasers [2]. Thin solid films of these materials have always been a subject of interest mainly because of their possible application to the manufacture of large-area photodiode arrays, solar selective coatings, solar cells, photoconductors, sensors and, so forth. PbSe thin film has been synthesized by various methods, including chemical bath deposition [3], vacuum deposition, sputtering, spray pyrolysis, electrodeposition [4], and microwave heating [5]. The doping process can significantly affect the physical properties of PbSe films, and it provides an easy way of changing the grain size and, thus, its emission and absorption wavelength, or optical band gap of the films [6-9].

Despite the significant progress which has taken place in coating technology, the deposition methods are difficult to control, and both the perfection of the layers and their bonding to the substrate can suffer failure. The possibility of introducing ion implantation, either as an additional step to enhance the bonding, or correct for deposition problems, can be exploited [10].
In the present work, we use physical vapor deposition (PVD) technique to synthesize a bilayer film from Sn layer over a PbSe substrate, followed by $\mathrm{Ar}^{+}$ion implantation. We compare the results obtained with different irradiation doses. It was found that it is possible to control the slight changes in optical band gap of PbSe by this process. Fourier transform infrared (FTIR) plots obtained from different samples showed the effects of Sn contents on the crystal quality, which is related to introduction of more defects induced by increasing the impurity concentration.

\section{Experimental Work}

PbSe layers were obtained by physical vapor deposition (PVD) from lead selenide powder on a glass substrate (microscope slide). High-purity $\mathrm{PbSe}$ powder (Aldrich \%99.999) and tin powder (Merc \%99.99) were used for preparing $\mathrm{Pb}_{1-x} \mathrm{Sn}_{x} \mathrm{Se} . \mathrm{Pb}_{1-x} \mathrm{Sn}_{x}$ Ses are prepared in a two-step process (by PVD) in which a thin PbSe layer $(\sim 300 \mathrm{~nm})$ is first deposited on a glass substrate, and then a very thin layer $(\sim 10 \mathrm{~nm})$ of $\mathrm{Sn}$ is deposited on top of the PbSe layer. Our attempt was to prevent the first layer to be oxidized before coating the second film on it. For this bilayer structure, we have a Sn/PbSe system. The thickness of the coated Sn atoms is sufficiently thin, that is, can be penetrated through the $\mathrm{PbSe}$ film by argon ion irradiation on top of the Sn layer. 


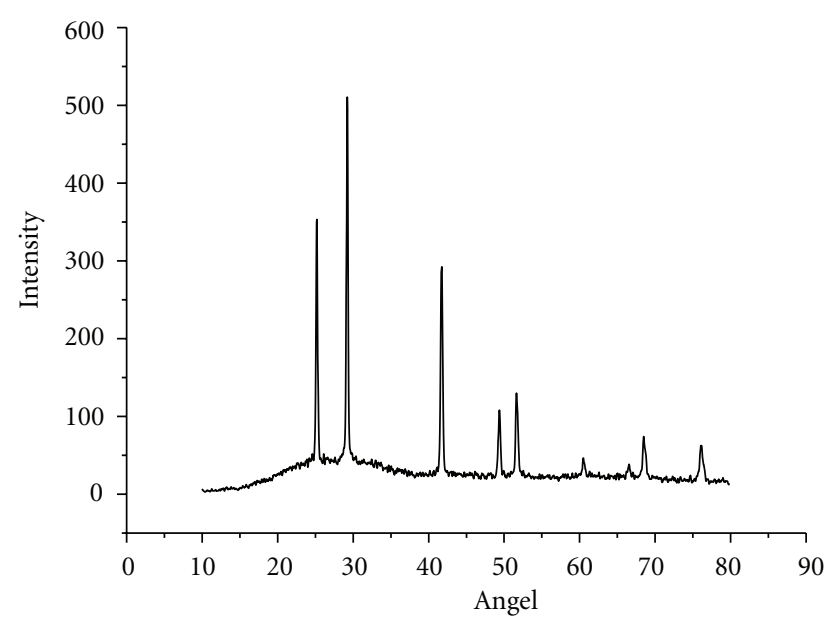

(a)

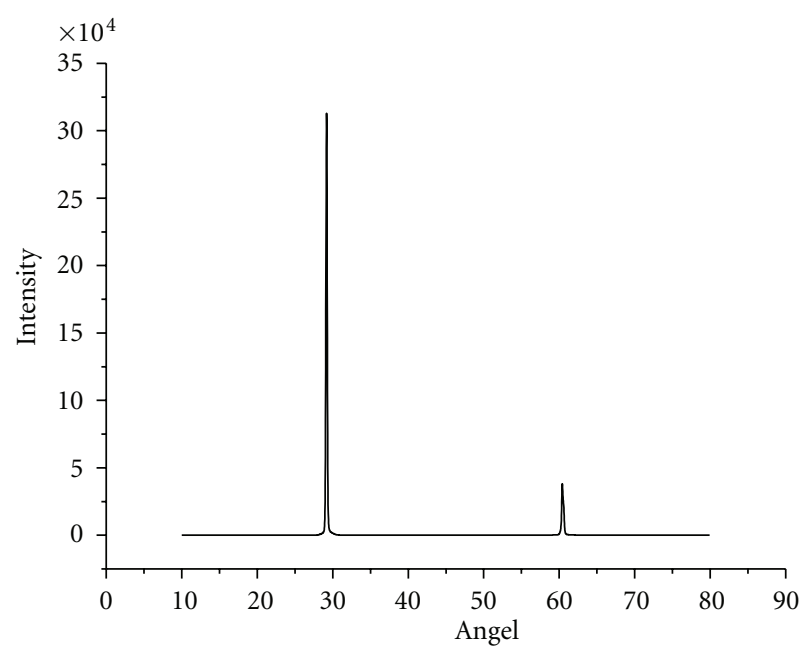

(b)

FIgure 1: XRD pattern from (a) as prepared $\mathrm{Sn} / \mathrm{PbSe}$ film and (b) ion-irradiated $\mathrm{Sn} / \mathrm{PbSe}$.

Ion implantation is especially good for the cases where the chemical or structural changes are desired to be near the surface of the target. The $\mathrm{Ar}^{+}$ion energy was fixed at $20 \mathrm{KeV}$ with three different doses of $10^{17}, 3 \times 10^{17}$, and $9 \times 10^{17} \mathrm{ion} / \mathrm{cm}^{2}$.

\section{Measurements}

3.1. X-Ray Diffraction. The crystal structure of PbSe incorporated with $\mathrm{Sn}$ was investigated using $\mathrm{X}$-ray $\mathrm{CuK} \alpha$ radiation at $\lambda=1.54 \AA$. The X-ray diffraction (XRD) pattern of (a) as prepared $\mathrm{Sn} / \mathrm{PbSe}$ film and (b) $\mathrm{Ar}^{+}$ion irradiated $\mathrm{Sn} / \mathrm{PbSe}$ are shown in Figure 1. Comparing nondoped PbSe (our unpublished work) to $\mathrm{Sn} / \mathrm{PbSe}$ reveals that, by adding $\mathrm{Sn}$ ions to PbSe matrix, a peak at $2 \theta \sim 25$ which is related to (111) Bragg plane of PbSe becomes higher than the peak at $2 \theta \sim 41$, from (220) plane, which for, PbSe alone, this relation is clearly inverse.

This can be due to a Bragg reflection, from SnSe crystal planes, which has a peak at $\sim 26$ and nothing at $\sim 41^{\circ}$.

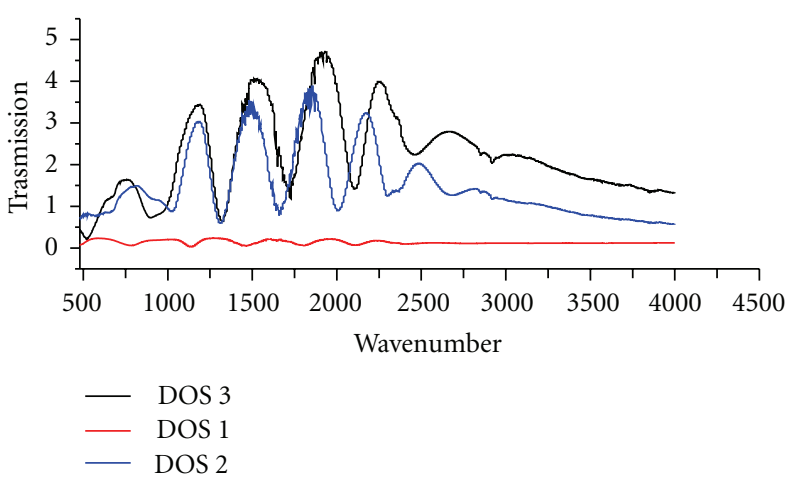

FIgURE 2: Transmission FTIR spectra from Sn-doped PbSe irradiated with three different doses.

TheXRD pattern of a typical SnSe thin film shows some features with more intense peaks at or around 26, 29 and also $51^{\circ}$. However, the preferred orientation of these films is at $2 \theta \sim 29$, which is attributed to (200) Bragg plane [11]. On the other hand, comparing the Figures 1(a)-1(b), we can observe that the main peak of PbSe film at $29^{\circ}$ is still the dominant one. However, other peaks except for a small feature at $2 \theta \sim 61$ have actually disappeared. It seems that ion implantation has a significant effect on the doped structure. Introduction of point defects in semiconductor materials, caused by ion implantation, is a common effect reported by many researchers [6-8]. Diffraction peaks for the irradiated sample (Figure 1(b)) are slightly sharper than the nonirradiated $\mathrm{Sn} / \mathrm{PbSe}$ and the full width at half maximum (FWHM) of the peak at $2 \theta=29$ related to the preferential plane; (200) is now very narrower $(\beta=0.154)$, indicating a relatively good crystal quality.

The crystallite grain size of the irradiated $\mathrm{Sn} / \mathrm{PbSe}$ calculated from the Debby-Scherer's formula:

$$
d=\frac{0.9 \lambda}{\beta \cos \theta},
$$

was approximately $53 \mathrm{~nm}$. Here, $\lambda$ and $\theta$ are the X-ray wavelength $(0.154 \mathrm{~nm})$ and the diffraction angle, respectively.

3.2. Optical Properties. FTIR transmission spectra for a ternary alloy; $\mathrm{Sn} / \mathrm{PbSe}$ layer irradiated by three different doses of $\mathrm{Ar}^{+}$ions are shown in Figure 2. Absorption edges around $10 \mu \mathrm{m}$ a dose of 3 (i.e., $9 \times 10^{17} \mathrm{ions} / \mathrm{cm}^{2}$ ) and $9.5 \mu \mathrm{m}$ for dose 2 (i.e., $3 \times 10^{17}$ ). These FTIR absorption edge spectra with the interference fringes agree very well with emission data from $\mathrm{Pb}_{1-x} \mathrm{Sn}_{x}$ Se alloys, having similar composition [9]. Almost equidistant spacing between the fringes are obtainable from $d v=1 / 2 n d$, in which $n$ and $d$, are refractive index and thickness of the films, respectively.

Sachar et al. [9] showed that the absorption edge decreases as tin content is increased. Comparing with the binary alloy of PbSe films, we can observe some more features in FTIR spectra which are not detected in the PbSe: Sn ternary alloy. Adding some more $\mathrm{Sn}$ atoms by increasing the dose, we can obtain up to $6 \%$ enhancement in full width half maximum (FWHM), extracted from the XRD system, 


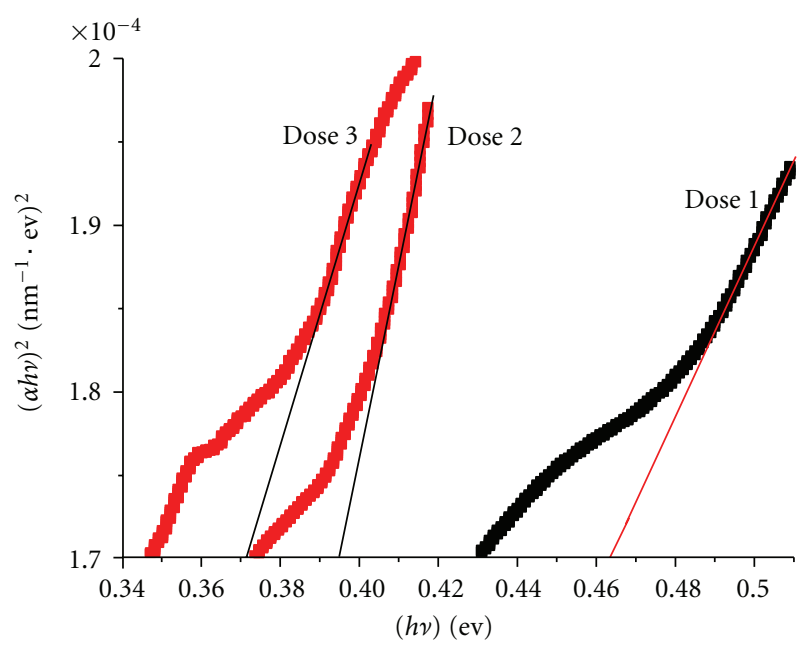

FIgURE 3: Variation of $(\alpha h \nu)^{2}$ with photon energy $(h \nu)$.

for a more concentrated film. This can be related to the alloying effect associated with the Sn incorporation which may deteriorate the crystal quality. Apart from a direct dopant concentration effect, the ion-beam bombardment could influence the process in other ways. The dose dependence of the optical transmission implies that its character is governed by ion-beam structure modification process. For the interaction of energetic particles with solids, the channeling effect is also important, and this effect could also play an important role in determining the dose dependence of the transmittance spectra. More studies are needed, however, to fully understand the interaction of the various mechanisms that may be involved during the transmission process in prepared doped PbSe, by this method.

For the present, work a direct transition was obtained, as shown in Figure 3, and the band gap for the films with different dose of irradiation named dose 1 to 3 was as follows; $0.462,0.395$, and $0.37 \mathrm{ev}$, respectively. The band gap energy is determined as the intersection of linear fits to the steeply declining transmission profile and the baseline above the transmission threshold.

As we mentioned above, under applied bombardment, the optical absorption edges shift towards lower energy and the direct band gaps of the alloy samples decrease. It has been indicated [12] that, in selenium-containing alloys, there is a tendency to form polymerization network glasses and the heteropolar bond of PbSe is qualitatively suppressed. This variation in band gap of these glassy films could be explained on the basis of bond formation between Se and $\mathrm{Sn}$. The addition of tin in PbSe system changes the configuration by $\mathrm{SnSe}_{2}$ tetrahedral phase and decreases the concentration of PbSe phase. Since $\mathrm{Sn}$ is added at the cost of $\mathrm{Pb}$ hence the concentration of $\mathrm{Se}-\mathrm{Sn}$ bonds (bond energy $401 \mathrm{~kJ} / \mathrm{mol}$ ) increases as compared to $\mathrm{Pb}-\mathrm{Se}$ bonds (bond energy $302 \mathrm{~kJ} / \mathrm{mol}$ ) [13] causing the decrease in band gap. Decrease in band gap for $\mathrm{Pb}_{1-x} \mathrm{Se} \mathrm{Sn}_{x}$ can also be explained on the basis of the model of density of states in amorphous solids proposed by Mott and Davis [14]. Note that by irradiation damage on PbSe network, some sharp peaks existed in nonirradiated XRD plot that disappeared in the irradiated one.

\section{Conclusion}

In summary, X-ray diffraction measurements were performed to study the effects of doping as well as ion implantation of lead selenide thin films prepared by physical vapor deposition. Irradiated $\mathrm{Sn} / \mathrm{PbSe}$ samples were found to exhibit a significant variation in structural response of the films by the Bragg plane diffraction. The optical band gap of $\mathrm{Sn} / \mathrm{PbSe}$ thin films was decreased by increasing the dose of irradiation.

\section{Acknowledgment}

The authors would like to express their thanks to Imam Khomeini International University for financial support.

\section{References}

[1] F. W. Wis, "Lead salt quantum dots: the limit of strong quantum confinement," Accounts of Chemical Research, vol. 33, no. 11, pp. 773-780, 2000.

[2] M. Böberl, W. Heiss, T. Schwarzl, K. Wiesauer, and G. Springholz, "Midinfrared continuous-wave photoluminescence of lead-salt structures up to temperatures of $190^{\circ} \mathrm{C}$," Applied Physics Letters, vol. 82, no. 23, pp. 4065-4067, 2003.

[3] G. Schmidt, "Large clusters and colloids. Metals in the embryonic state," Chemical Reviews, vol. 92, no. 8, pp. 1709-1727, 1992.

[4] A. N. Molin and A. I. Dikusar, "Electrochemical deposition of PbSe thin films from aqueous solutions," Thin Solid Films, vol. 265, no. 1-2, pp. 3-9, 1995.

[5] J. Zhu, O. Palchik, S. Chen, and A. Gedanken, "Microwave assisted preparation of $\mathrm{CdSe}, \mathrm{PbSe}$, and $\mathrm{Cu}_{2-x} \mathrm{Se}$ nanoparticles," Journal of Physical Chemistry B, vol. 104, no. 31, pp. 7344-7347, 2000.

[6] D. P. Joseph, P. Renugambal, M. Saravanan, S. P. Raja, and C. Venkateswaran, "Effect of Li doping on the structural, optical and electrical properties of spray deposited $\mathrm{SnO}_{2}$ thin films," Thin Solid Films, vol. 517, no. 21, pp. 6129-6136, 2009.

[7] P. D. Townsend, M. Khanlary, and D. E. Hole, "Information obtainable from ion beam luminescence," Surface and Coatings Technology, vol. 201, no. 19-20, pp. 8160-8164, 2007.

[8] L. J. Li, M. Glerup, A. N. Khlobystov et al., "The effects of nitrogen and boron doping on the optical emission and diameters of single-walled carbon nanotubes," Carbon, vol. 44, no. 13, pp. 2752-2757, 2006.

[9] H. K. Sachar, I. Chao, P. J. McCann, and X. M. Fang, "Growth and characterization of $\mathrm{PbSe}$ and $\mathrm{Pb}_{1-x} \mathrm{Sn}_{x} \mathrm{Se}$ on Si (100)," Journal of Applied Physics, vol. 85, no. 10, pp. 7398-7403, 1999.

[10] M. Khanlary, P. Townsend, B. Ullrich, and D. E. Hole, "Ionbeam luminescence of thin-film CdS on glass formed by pulsed-laser deposition," Journal of Applied Physics, vol. 97, no. 2, Article ID 023512, 2005.

[11] N. A. Okereke and A. J. Ekpunobi, "Structure and optical properties of chemically deposited tin selenide," Chalcogenide Letters, vol. 7, no. 9, pp. 531-538, 2010.

[12] M. Rabinal, N. Ramesh Rao, and K. Sanngunni, "Non-isothermal transformation of Pb-modified Ge-Se glasses," Philosophical Magazine, vol. 70, no. 1, pp. 89-99, 1994. 
[13] R. David, CRC Handbook of Chemistry and Physics, Chemical Rubber, Cleveland, Ohio, USA, 1997.

[14] N. Mott and E. Davis, Electric Processes in Non Crystalline Materials, Oxford, UK, 1971. 

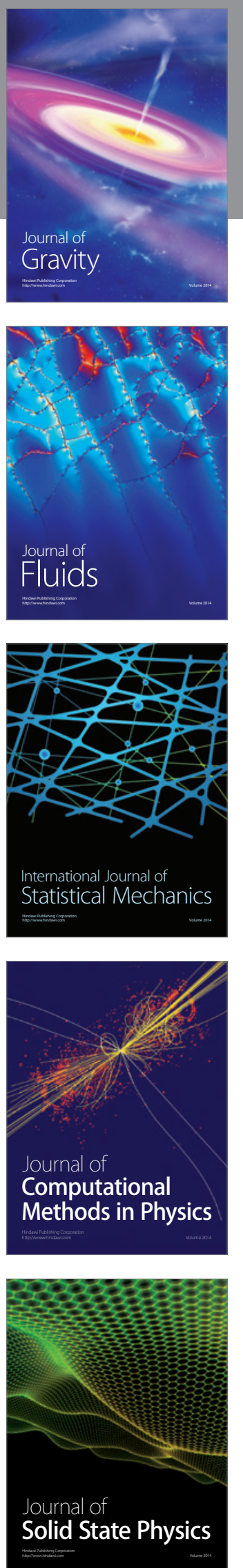

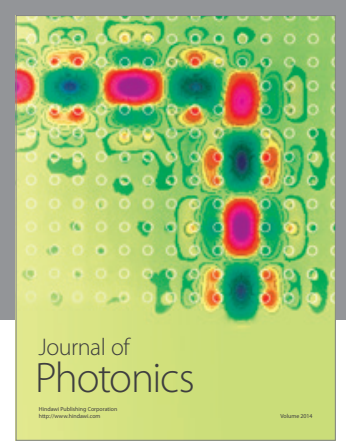

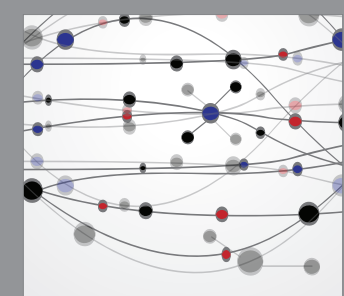

The Scientific World Journal
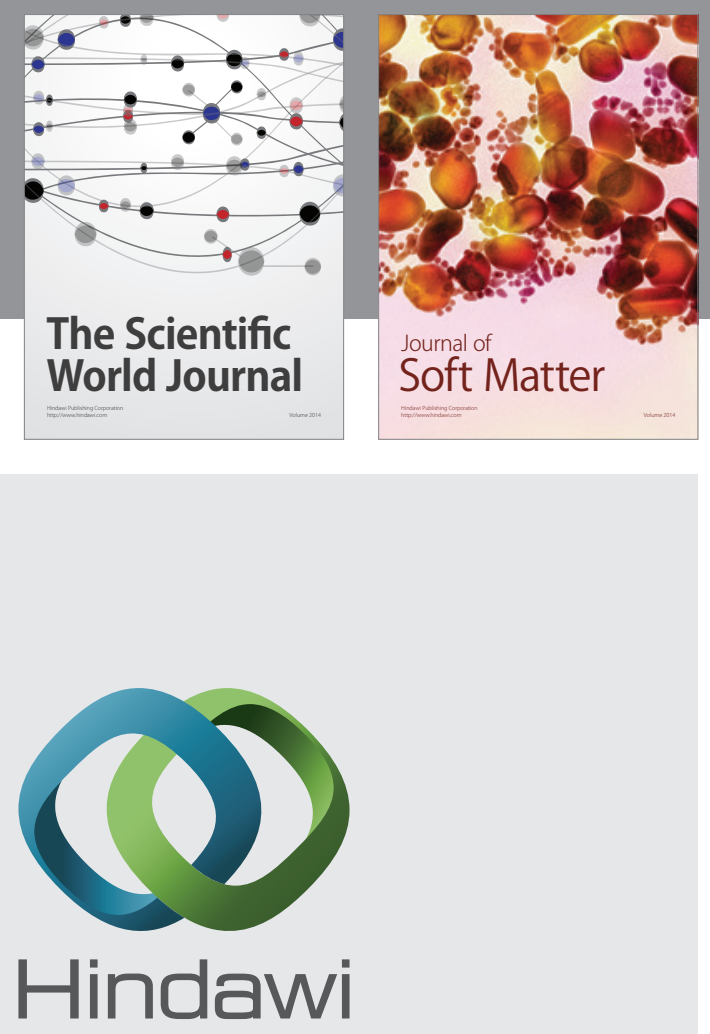

Submit your manuscripts at

http://www.hindawi.com
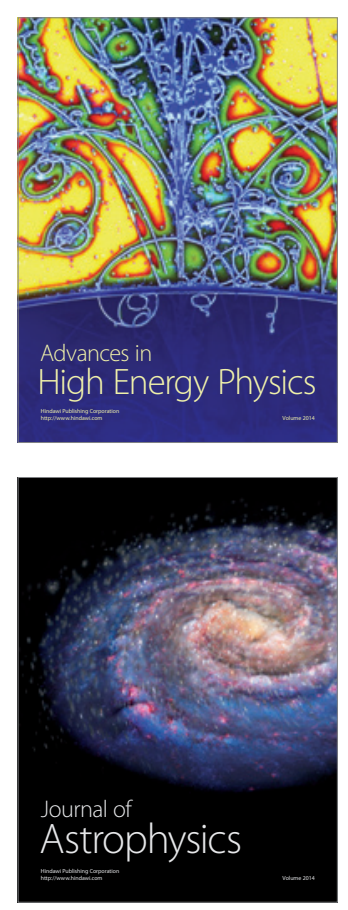
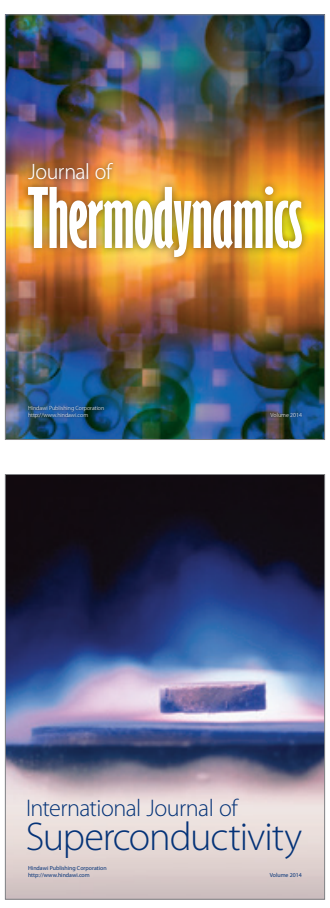
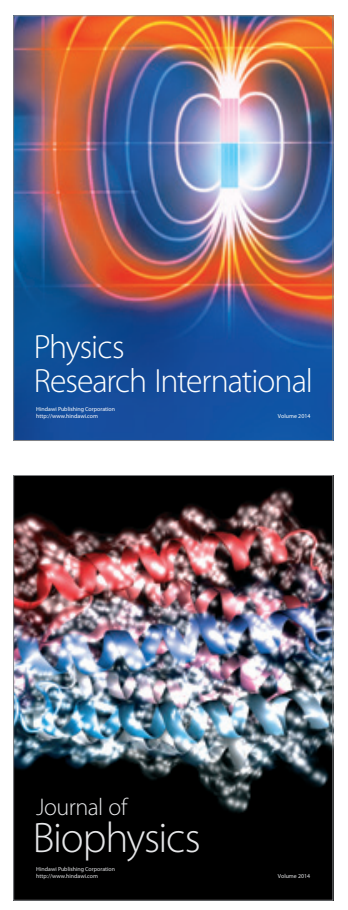
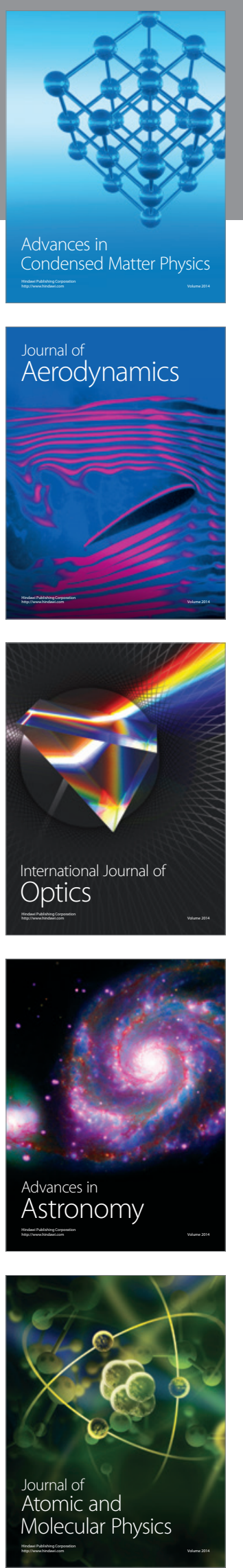